\title{
The in vitro rabbit whole bladder as a model to investigate the urothelial transport of anticancer agents The ONCOFID- $\mathrm{P}^{\circledR}$ paradigm
}

\author{
Giuseppe Tringali ${ }^{\mathrm{a}}$, Lucia Lisi ${ }^{\mathrm{a}}$, Fabio Bettella ${ }^{\mathrm{b}}$, \\ Davide Renier ${ }^{\mathrm{b}}$, Savino M. Di Stasi ${ }^{\mathrm{c}}$, Pierluigi Navarra ${ }^{\mathrm{a}, *}$ \\ a Institute of Pharmacology, Catholic University Medical School, Largo Francesco Vito 1, 00168 Rome, Italy \\ b Fidia Farmaceutici, Abano Terme, Italy \\ ' Department of Urology, 'Tor Vergata' University of Rome, Rome, Italy
}

\section{A R T I C L E I N F O}

Article history:

Accepted 12 September 2008

\section{Keywords:}

Bladder

Hyaluronic acid

Intravesical route

Paclitaxel

rabbit

Transport

Urothelium

\begin{abstract}
A B S T R A C T
Intravesical instillation of BCG or anticancer agents after transurethral resection is currently considered a standard of therapy. However, this approach is basically empirical; none of the anticancer agents used in this setting was specifically formulated for intravesical therapy. Moreover, concern is raised by the kinetic features of water soluble drugs, because of poor transport across the mucosal barrier, or of liphophylic compounds, for the increased risks of systemic toxicity. A need exists to improve the pre-clinical and clinical approaches used at present to test anticancer agents undergoing specific development for intravesical use. We used in vitro rabbit whole bladders as a new pre-clinical model to investigate the kinetics of locally administered anticancer agents. In this study, we investigated the rate of urothelial transport of a novel paclitaxel derivative, Oncofid- $\mathrm{P}^{\circledR}$.

Male New Zealand albino rabbits were used. Bladders were rapidly explanted, filled with vehicle alone or vehicle containing graded concentrations of Oncofid-P, and kept for various times under standardized incubation conditions. At the end of experiments, drug concentrations were assessed by high-pressure-liquid-chromatography technique in the intravesical and external bath solutions, as well as in bladder wall homogenates. We found that less than $1 \%$ of the drug additioned to the intravesical solution is recovered within the bladder wall in the form of paclitaxel; experiments carried out collecting different areas from the same bladders showed that Oncofid-P is uniformly distributed over the internal surface of bladder mucosa. Isolated rabbit bladders may be a useful pre-clinical model to investigate the rate of transport of chemotherapeutic agents administered by intravesical route. In this paradigm, Oncofid-P displays a kinetic profile that is predictive of local activity over the whole urothelial surface, and low or absent systemic absorption.
\end{abstract}

(c) 2008 Elsevier Ltd. All rights reserved.

\section{Introduction}

Transurethral resection (TUR) is the routine initial diagnostic and therapeutic step in management of non-muscle invasive bladder cancer. Recurrence rate following TUR alone occurs on average in $45 \%$ of patients within 1 year, and progression to muscle invasive and/or metastatic bladder cancer are reported as varying from $3 \%$ to $15 \%$ of cases [1]. The probability of disease recurrence and progression have led to widespread use of intravesical therapies with cytotoxic and immunotherapeutic agents [1-3]. A recent

* Corresponding author. Tel.: +39 06 30154253/4367/4531; fax: +3906233235103

E-mail address: pnavarra@rm.unicatt.it (P. Navarra). meta-analysis shows that, compared with patients treated by TUR alone, those receiving adjuvant intravesical mitomycin-C (MMC) or bacillus Calmette-Guerin (BCG) have a reduced incidence of tumor recurrence, even if the ultimate long term benefits are in doubt [1].

Notwithstanding the modest outcomes on hard endpoints, intravesical therapy became by the time a standard of care. However, drawbacks exist that must be taken in due account. First, this approach has been developed essentially on an empirical basis, and should be considered as an off-label use. One important pitfall of this situation is that none of the anticancer agents currently used for this purpose has been specifically formulated for intravesical therapy; according to the current practice, injectable solutions intended for parenteral administration are diluted in appropriate volumes of solvents (normal saline, sterile distilled water) and instilled into the 
bladder. Such lack of standardization might have negative reflections on drug absorption [4].

Second, while water soluble drugs such as MMC face the problem of scarce diffusion through the urothelial barrier [4,5], on the opposite highly lipophylic compounds are associated to the potential risk of increased systemic absorption. Therefore, for anticancer agents that are under development for intravesical use, it is mandatory today that adequate pre-clinical and clinical testing are carried out before registration, to demonstrate efficacy and safety of local intravesical treatments; moreover, special attention should be paid to the physico-chemical features of the candidate drug.

The rabbit whole bladder in vitro model has been widely used in the past for functional or pharmacological studies on smooth muscle contractility [6,7] or urothelial permeability [8], as well as a model of human bladder for morphological investigations $[9,10]$. This is the first study to our knowledge where isolated rabbit bladders are used to investigate the kinetics of locally administered anticancer agents. In particular, here we investigated the rate of urothelial transport of a novel paclitaxel derivative, Oncofid- ${ }^{\circledR}$ [11], which is currently under development for intravesical use in patients with non-muscle invasive bladder cancer.

\section{Materials and methods}

\subsection{Experimental procedure}

The use of animals for this experimental work has been approved by Italian Ministry of Health (licensed authorization to Prof. P. Preziosi). Male New Zealand albino rabbits, weighting approximately $2.5-3 \mathrm{Kg}$ were used. On the day of experiment, the animals were killed with a lethal dose of anaesthetic, $50 \mathrm{mg} / \mathrm{kg}$ pentothal sodium by intra-peritoneal route. The urinary bladder, urethra and ureters were exposed through midline incision and subsequently cleared from fat and connective tissue. Both ureters were tied and the whole bladder was rapidly removed from the base at the level of the ureteral orifices. The urinary bladder was emptied, washed with Krebs solution and filled with 5\% glucose solution alone (control) or 5\% glucose solution containing 7.5 or $15 \mathrm{mg} / \mathrm{ml}$ ONCOFID-P (equivalent to $1.5 \mathrm{mg}$ or $3 \mathrm{mg} / \mathrm{ml}$ paclitaxel, respectively). Subsequently, bladders were incubated in $200 \mathrm{ml}$ Krebs bubbled with a $95 \% \mathrm{O}_{2} / 5 \% \mathrm{CO}_{2}$ mixture, at $37^{\circ} \mathrm{C}$ in a Dubnoff shaking bath. At the end of experiments, bladders were emptied, washed and weighted. Intravesical solutions, washing solutions and external bath solutions were collected and stored at $-80^{\circ} \mathrm{C}$ until assay for paclitaxel by high-performance liquid chromatography (HPLC) technique.

In experiments performed to investigate uniform distribution of the drug over the internal surface, bladders were opened and flattened on a Petri dish plane; thereafter, four different points of the bladder wall, located at the corners of a virtual square traced on the surface of bladder surface (conventionally north, south, east and west) were selected, tissue fragments were dissected, homogenated and stored as above described for subsequent HPLC analysis.

In order to measure paclitaxel into the tissues, bladders were snap-frozen and kept at $-80^{\circ} \mathrm{C}$ until homogenization. The latter was performed in $5 \%$ glucose solution using a Teflon glass homogenizer (DuPont Co., Wilmington, DE). Supernatants were collected and stored at $-80^{\circ} \mathrm{C}$.

\subsection{Experimental procedure validation}

Preliminary studies were carried out to validate the method and optimize the experimental conditions. To this purpose, the following series of experiments were conducted: (i) with different filling volumes of $5 \%$ glucosate $(10 \mathrm{ml}, n=2$ in 1 experimental session; $25 \mathrm{ml}, n=4$ in 2 experimental sessions; $50 \mathrm{ml}, n=21$ in 5 experimental sessions; $75 \mathrm{ml}, n=6$ in 2 experimental sessions; $100 \mathrm{ml}, n=2$ in 1 experimental session); from these experiments, $100 \mathrm{ml}$ resulted to be a supra-maximal filling volume, because of extravasation of the internal solution, whereas $50 \mathrm{ml}$ were taken as average bladder capacity;

(ii) with graded ONCOFID-P concentrations $(0.5 \mathrm{mg} / \mathrm{ml}, 5,15$ and $25 \mathrm{mg} / \mathrm{ml} ; n=2$ for all concentrations) at fixed filling volume $(50 \mathrm{ml})$ and incubation time $(1 \mathrm{~h})$;

(iii) with graded incubation times $(15,30,60$ or $120 \mathrm{~min})$.

In all of these experiments, the amount of ONCOFID-P recovered in the intravesical bath solutions was always included within 97-99\% of the initial dose instilled in the bladder.

\subsection{Analytical method}

An HPLC assay was developed to estimate free paclitaxel. Such assay did not allow to detect and measure paclitexel bound to hyaluronic acid (HA); therefore, prior to HPLC analysis, samples had to be submitted to basic hydrolysis with $0.1 \mathrm{M} \mathrm{NaOH}$ at $30^{\circ} \mathrm{C}$ for $1 \mathrm{~h}$ in order to cleave paclitaxel from binding to HA. Thereafter samples of intravesical, washing and external bath solutions were neutralized with $0.1 \mathrm{M} \mathrm{HCl}$ and diluted with a mixture of absolute $\mathrm{EtOH} / \mathrm{H}_{2} \mathrm{O}(60 / 40, \mathrm{v} / \mathrm{v})$. After neutralization, homogenate samples were extracted with diethyl ether and the mixture fully evaporated $\left(\mathrm{N}_{2}\right.$ flow at $\left.30-35^{\circ} \mathrm{C}\right)$ and diluted in acetonitrile/ $\mathrm{H}_{2} \mathrm{O}(50 / 50$, $\mathrm{v} / \mathrm{v})$. The above procedure allows to measure and express the total amount of paclitaxel in each sample (i.e. the free fraction plus that bound to HA) as free paclitaxel.

HPLC analysis was performed on a PerkinElmer series 200 instrument (Shelton, CT), with UV detection at $254 \mathrm{~nm}$. Nova-Pak C18 Waters $(15 \times 3.9 \mathrm{~mm})$ columns (Milford, MA) were used, and the eluent was acetonitrile/water $(44 / 56, v / v)$, with a flow rate of $1.0 \mathrm{ml} / \mathrm{min}$.

\subsection{Chemicals and drugs}

ONCOFID-P was kindly provided by Fidia Farmacetici spa (Abano Terme, Italy). Two different batches of ONCOFID-P were used: RS118/04 (degree of esterification equal to $21.77 \% \mathrm{w} / \mathrm{w}$ of the dry compound and loss on drying of 3.55\%); $05 R 0001$ (degree of esterification equal to $21.6 \% \mathrm{w} / \mathrm{w}$ of the dry compound and loss on drying of $6.4 \%$ )

ONCOFID-P was dissolved and diluted to working concentrations in $5 \%$ glucose solution. All other reagents were obtained from Carlo Erba (Milan, Italy).

\subsection{Statistical analysis}

Experiments were carried in duplicate or in triplicate, according to a randomized block design. Data were expressed as means \pm standard deviation (S.D.) of six or ten replicates for experimental group. Statistical analysis were carried out using one-way ANOVA followed by post-hoc Newman-Keuls test for multiple comparison among group means, using a Prism computer program (GraphPad, San Diego, California, USA); differences were taken as significant if $P<0.05$.

\section{Results}

After method validation, conducted as described in the Section 2 , a series of experiments was carried out with fixed incubation times ( $1 \mathrm{~h}$, i.e. the timing usually adopted in clinical practice) and 


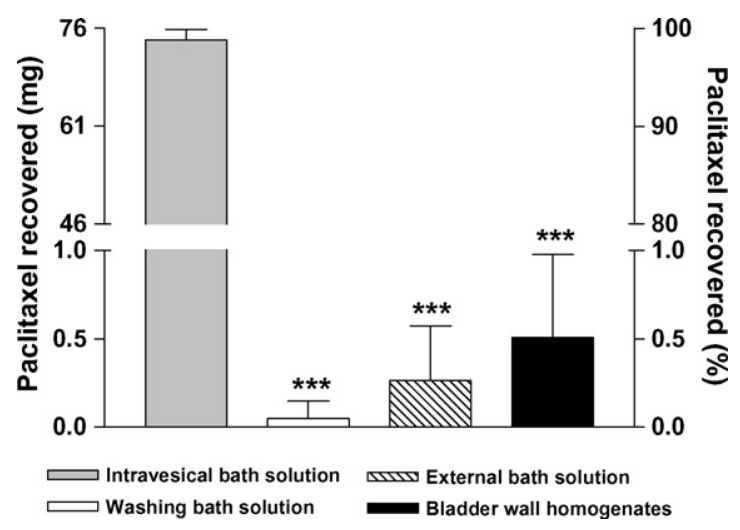

Fig. 1. Paclitaxel recovered in the intravesical, washing and external bath solutions, as well as into the bladder tissue. The total dose administered was $75 \mathrm{mg}$ Paclitaxel $/ 25 \mathrm{ml}$. Results are the means \pm S.D. of $\mathrm{mg}$ of native paclitaxel (left $Y$-axis) or as the percent of the dose (right $Y$-axis). Three independent experiments were conducted (randomized block design); data from the three experiments were pooled to obtain a total of 10 replicates per experimental group. ${ }^{* * *}: p<0.001$ vs. intravesical bath solution.

filling volumes ( $25 \mathrm{ml}$ of $5 \%$ glucosate, i.e. a sub-maximal filling volume, as in the clinical setting), using a Paclitaxel concentration of $3 \mathrm{mg} / \mathrm{ml}$ [corresponding to $342 \mathrm{mg} / 25 \mathrm{ml}$ of ONCOFID-P (RS118/04) or to $351 \mathrm{mg} / 25 \mathrm{ml}$ of ONCOFID-P (05R0001), depending on the different degree of esterification of the two batches]. In these experiments, we carried out simultaneous determinations of paclitaxel in the intravesical and extra-vesical bath solutions, as well as within the bladder wall tissue. Consistent with the data of preliminary experiments, we found that paclitaxel was almost completely recovered in the intravesical bath solution $(98.9 \%$ on average), whereas $0.68 \%$ of the dose was detected within the bladder wall and $0.42 \%$ in the external bath solution (Fig. 1 ). Similar results were obtained with paclitaxel given at the dose of $1 \mathrm{mg} / \mathrm{ml}$ (data not shown)

Further experiments were performed to investigate whether paclitaxel is uniformly distributed throughout the internal bladder surface. Similar to previous experiments, tiny amounts of paclitaxel were recovered within the bladder wall (approximately $0.2 \%$ ). No significant differences in paclitaxel levels were observed among the four different areas of bladder wall, indicating that the drug is uniformly distributed within the tissue (Fig. 2).

\section{Discussion}

In this study we used isolated rabbit bladders to investigate the rate of transport of Oncofid-P across the urothelium. The main findings of the study are the following: (i) less than $1 \%$ of the administered drug is recovered within the bladder wall in the form of paclitaxel; (ii) experiments carried out collecting different areas from the same bladders show that Oncofid-P is uniformly distributed in the bladder mucosa. It is clear from our findings that the esterification of paclitaxel with HA makes the pharmacokinetics of the complex similar to those of other more hydrophilic drugs, such as MMC and doxorubicin, which display a rate of absorption $<5 \%$ of the concentration in urine [12]. Esterification with HA also appeared to sort out another problem of native paclitaxel, i.e. precipitation into micelles. Thus, Oncofid-P possesses interesting potential advantages over paclitaxel.

The latter has been envisioned as a better alternative to MMC in treating non-muscle invasive bladder cancers, since its higher lipo-solubility should facilitate penetration across the urothelium and accumulation within the bladder wall [12]. On these basis, a number of pre-clinical in vitro and in vivo studies were car-

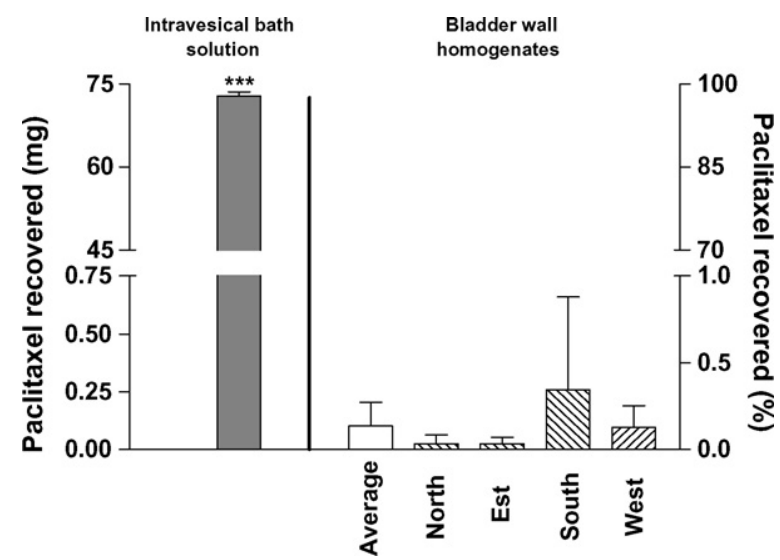

Fig. 2. Paclitaxel recovered in intravesical bath solution (full Bar) and in selected areas (conventionally North, East, South, West) of the bladder wall (dashed bars). The empty bar shows the average amount of the four selected parts. The total dose administered was $75 \mathrm{mg}$ Paclitaxel $/ 25 \mathrm{ml}$. Results are the means \pm S.D. of $\mathrm{mg}$ of native paclitaxel (left $Y$-axis) or as the percent of the dose (right $Y$-axis). Two independent experiments were conducted (randomized block design); data from the two experiments were pooled to obtain a total of six replicates per experimental group. ${ }^{* * *}: p<0.001$ vs. the average of $\mathrm{N}, \mathrm{E}, \mathrm{S}$ and $\mathrm{W}$.

ried out, and paclitaxel was found effective as an anti-proliferative agent in different paradigms, suggesting its potential usefulness in the clinical setting [13-15]. However, standard paclitaxel formulations are currently prepared in Cremophor, a mixture of castor oil and ethanol, to be diluted in water solutions before administration; Cremophor in urine tends to form micelles which entrap paclitaxel, thereby greatly reducing the potential advantages brought about by paclitaxel lipo-solubility [16,17]. In order to overcome this problem, different formulations of paclitaxel have been developed and investigated, including the addition of dimethyl sulfoxide, the inclusion of paclitaxel in bio-adhesive polymer micro-spheres or in gelatine nanoparticles, and the use of drug delivery systems with glycerol monooleate [17-20]. All these efforts testify a huge and diffuse interest in developing suitable formulations for effective intravesical delivery of paclitaxel.

Oncofid-P, formerly referred to as Hytad1-p20, is a new hydro-soluble formulation of paclitaxel, obtained through bioconjugation with HA [11]. Such esterification process gives rise to a sort of pro-drug formulation, which releases free paclitaxel after bodily hydrolysis of the ester. Apart from higher water solubility, the HA component of the molecule binds with high affinity to membrane CD44, which is over expressed in several tumor types, including bladder cancer; CD44 binding should potentially increase Oncofid-P concentrations within the target tumor tissues.

Apart from binding to tumor cells, the questions arises as to whether Oncofid-P binding to CD44 also plays a role in the passage across urothelium; indeed, adhesion to CD44 on the superficial cell layer may represent the first step of the absorption process. CD44 was reported to be expressed and produced in the basal layers of normal human urothelium, but expression is lost as epithelial cells progress from the basal to the superficial layer [21]. The same pattern of CD44 expression has been described in rabbit bladder [22]; in both studies, the standard CD44 isoform (CD44s) was investigated. Thus, the scarce, if any, expression of CD44 s at the superficial layers of rabbit urothelium might account for the low amounts of Oncofid-P uptaken by the mucosa in the present study. As also explained in other paragraphs, this phenomenon turns out to be a potential advantage in the therapeutic setting, since it maintains the drug confined at the site of action in the bladder lumen, while 
on the same time reducing the risks of toxicity related to systemic absorption.

CD44 gene structure, with 10 constant and 10 variable exons, accounts for more than 20 isoforms, with molecular weights ranging from 85 to $230 \mathrm{kDa}$. A CD44 isoform containing the last three exon products of the variable region, also known as epithelial CD44 or CD44e, is preferentially expressed on epithelial cells [23]. The expression and production of epithelial CD44 in the rabbit urothelium has not been investigated. Many cancer cell types, including bladder cancer, may express CD44 variants [23]. A few studies have been conducted thus far to address the issue of HA binding to CD44 variants; at least in the case of human peripheral blood T cells, HA displays a higher binding affinity toward splice variants compared to the standard form [24].

The rabbit whole bladder in vitro model has been used here for the first time to investigate kinetic parameters of an anticancer agent given by the intravesical route. Such novel usage deserves specific discussion. This paradigm shares with other in vitro models the advantage of excluding the influence of confounds such as blood and urine flow, which increase variability of the in vivo models $[25,26]$. On the other side, while allowing a more accurate experimental assessment, all of the techniques that exclude blood and urine flow in some way increase their distance from the clinical setting. Another advantage of using rabbit bladders lies on less technical (availability of samples, transport of bladder tissue) and perhaps ethical problems with respect to the use of dogs, pigs or else human bladders from surgical specimens. Moreover, the use of whole bladders instead of fragments however large, allows a better assessment of drug diffusion over the whole surface of bladder mucosa.

As far as the present study is concerned, two technical points need to be addressed. First, the analytical method adopted in this study allows to measure native paclitaxel, but not the complex paclitaxel-HA; therefore, the complex requires to be hydrolysed before analysis, as described in the Methods section. Because of this limitation, we could not address the question as to whether paclitaxel recovered within the bladder wall was in the form of ester, or it was hydrolysed by tissue esterases, or both. Likewise, we could not ascertain whether Oncofid-P undergoes hydrolysis before being absorbed. However, we have previously demonstrated that Oncofid-P is stable in human urines at pH 6.5 throughout 6 h of incubation at $37^{\circ} \mathrm{C}[11]$; therefore, it is likely that Oncofid-P remains stable in our paradigm throughout the duration of $1 \mathrm{~h}$ experiments, and therefore it is absorbed as such. This hypothesis is consistent with the low rate of delivery observed here, which is suggestive of a relatively high hydro-solubility.

Second, levels of paclitaxel in the same order of magnitude of that found within the bladder wall were detected in the external bath solution. Based on this finding, one might hypothesize that the drug may undergo a passage through the entire thickness of the bladder wall. While this hypothesis cannot be ruled out, it is in sharp contrast with the low rate of absorption from the intravesical bath solution to the bladder wall. An alternative, more reasonable, explanation would be that tiny amounts of drug spill over from the sites of bladder ligature (urethra and ureters) during the experiments, because of intravesical pressure and shaking bath movements. Contamination of the external bladder surface caused by the manipulations preceding the incubation step may also play a role.

In conclusion, isolated rabbit bladders proved to be a useful pre-clinical model to investigate the rate of transport of chemotherapeutic agents administered by intravesical route. In this paradigm, Oncofid-P displays a kinetic profile that is predictive of local activity over the whole urothelial surface, and low or absent systemic absorption.

\section{Acknowledgements}

This work has been supported by a grant from Fidia Farmaceutici, Abano Terme, Italy.

We kindly acknowledge Mr. Giuseppe Ripanti for expert technical assistance.

\section{References}

[1] Hall MC, Chang SS, Dalbagni G, Pruthi RS, Seigne JD, Skinner EC, et al. Guideline for the management of nonmuscle invasive bladder cancer (stages Ta, T1, and Tis): 2007 update. J Urol 2007;178(6):2314-30.

[2] Witjes JA, Hendricksen K. Intravesical pharmacotherapy for nonmuscle-invasive bladder cancer: a critical analysis of currently available drugs, treatment schedules, and long-term results. Eur Urol 2008;53: 45-52.

[3] Di Stasi SM, Giannantoni A, Stephen RL, Capelli G, Navarra P, Massoud R, et al Intravesical electromotive mitomycin $C$ versus passive transport mitomycin $C$ for high risk superficial bladder cancer: a prospective randomized study. J Urol 2003;170:777-82.

[4] Di Stasi SM, Giannantoni A, Massoud R, Dolci S, Navarra P, Vespasiani G, et al. Electromotive versus passive diffusion of mitomycin $C$ into human bladder wall: concentration-depth profiles studies. Cancer Res 1999;59:4912-8.

[5] Di Stasi SM, Vespasiani G, Giannantoni A, Massoud R, Dolci S, Micali F. Electromotive delivery of mitomycin $C$ into human bladder wall. Cancer Res 1997:57:875-80.

[6] Kato K, Kitada S, Chun A, Wein AJ, Levin RM. In vitro intravesical instillation of anticholinergic, antispasmodic and calcium blocking agents (rabbit whole bladder model). J Urol 1989;141:1471-5.

[7] Matsumoto S, Chichester P, Bratslavsky G, Kogan BA, Levin RM. The functional and structural response to distension of the rabbit whole bladder in vitro. J Urol 2002;168:2677-81.

[8] Tammela T, Wein AJ, Monson FC, Levin RM. Urothelial permeability of the isolated whole bladder. Neurourol Urodyn 1993;12:39-47.

[9] Agartan CA, Whitbeck C, Chichester P, Kogan BA, Levin RM. Effect of age on rabbit bladder function and structure following partial outlet obstruction. J Urol 2005; 173:1400-5.

[10] Landau EH, Fung LC, Thorner PS, Mittelman MW, Jayanthi VR, Churchill $\mathrm{BM}$, et al. Histologic studies of intravesical oxybutynin in the rabbit. J Urol 1995; 153:2022-4.

[11] Rosato A, Banzato A, De Luca G, Renier D, Bettella F, Pagano C, et al. HYTAD1p20: a new paclitaxel-hyaluronic acid hydrosoluble bioconjugate for treatment of superficial bladder cancer. Urol Oncol 2006;24:207-15.

[12] Song D, Wientjes MG, Au JL. Bladder tissue pharmacokinetics of intravesica taxol. Cancer Chemother Pharmacol 1997;40:285-92.

[13] Rangel C, Niell H, Miller A, Cox C. Taxol and taxotere in bladder cancer: in vitro activity and urine stability. Cancer Chemother Pharmacol 1994;33:460-4.

[14] Au JL, Kalns J, Gan Y, Wientjes MG. Pharmacologic effects of paclitaxel in human bladder tumors. Cancer Chemother Pharmacol 1997;41:69-74.

[15] Nativ O, Aronson M, Medalia O, Moldavsky T, Sabo E, Ringel I, et al. Antineoplastic activity of paclitaxel on experimental superficial bladder cancer: in vivo and in vitro studies. Int J Cancer 1997;70:297-301.

[16] Knemeyer I, Wientjes MG, Au JL. Cremophor reduces paclitaxel penetration into bladder wall during intravesical treatment. Cancer Chemother Pharmacol 1999;44:241-8.

[17] Chen D, Song D, Wientjes MG, Au JL. Effect of dimethyl sulfoxide on bladder tissue penetration of intravesical paclitaxel. Clin Cancer Res 2003;9: 363-9.

[18] Le Visage C, Rioux-Leclercq N, Haller M, Breton P, Malavaud B, Leong K. Efficacy of paclitaxel released from bio-adhesive polymer microspheres on model superficial bladder cancer. J Urol 2004;171:1324-9.

[19] Lu Z, Yeh TK, Tsai M, Au JL, Wientjes MG. Paclitaxel-loaded gelatin nanoparticles for intravesical bladder cancer therapy. Clin Cancer Res 2004;10:7677-84

[20] Lee SJ, Kim SW, Chung H, Park YT, Choi YW, Cho YH, et al. Bioadhesive drug delivery system using glyceryl monooleate for the intravesical administration of paclitaxel. Chemotherapy 2005;51:311-8.

[21] Southgate J, Trejdosiewicz LK, Smith B, Selby PJ. Patterns of splice variant CD44 expression by normal human urothelium in situ and in vitro and by bladdercarcinoma cell lines. Int J Cancer 1995;62:449-56.

[22] Arafat HA, Wein AJ, Chacko S. Osteopontin gene expression and immunolocalization in the rabbit urinary tract. J Urol 2002;167:746-52.

[23] Naor D, Sionov RV, Ish-Shalom D. CD44: structure, function, and association with the malignant process. Adv Cancer Res 1997;71:241-319.

[24] Galluzzo E, Albi N, Fiorucci S, Merigiola C, Ruggeri L, Tosti A, et al. Involvement of CD44 variant isoforms in hyaluronate adhesion by human activated T cells. Eur J Immunol 1995;25:2932-9.

[25] Wientjes MG, Badalament RA, Wang RC, Hassan F, Au JL. Penetration of mitomycin C in human bladder. Cancer Res 1993;53:3314-20.

[26] Wientjes MG, Dalton JT, Badalament RA, Drago JR, Au JL. Bladder wall penetration of intravesical mitomycin C in dogs. Cancer Res 1991;51:4347-54. 